\title{
A double-blinded, randomized, placebo-controlled study evaluating the impact of dates vinegar consumption on blood biochemical and hematological parameters in patients with type 2 diabetes
}

\author{
Zeshan Ali ${ }^{1 \star}$, Haile Ma ${ }^{1}$, Asif Wali ${ }^{2}$, Ishmael Ayim¹, Muhammad Tayyab Rashid", \\ Shoaib Younas ${ }^{3}$ \\ ${ }^{1}$ School of Food and Biological Engineering, Jiangsu University, Zhenjiang 212013, China \\ ${ }^{2}$ Department of Agriculture and Food Technology, Karakoram International University, Gilgit 15100, \\ Pakistan, ${ }^{3}$ Department of Food Science and Nutrition, University of Sargodha, Pakistan
}

${ }^{*}$ For correspondence: Email: mhl@ujs.edu.cn

Revised accepted: 11 September 2018

\begin{abstract}
Purpose: To determine the effects of dates vinegar on blood biochemical and hematological parameters in type 2 diabetic subjects.

Methods: Current research focused on fifty-five subjects having blood sugar more than126 mg/dL. Participants ingested dates vinegar $(20 \mathrm{~mL})$ daily into their normal diets for a period of 10 weeks. Glycated hemoglobin (HbA1c), fasting blood sugar (FBS), total cholesterol, high-density lipoprotein $(H D L)$, low-density lipoprotein ( $L D L)$, creatinine (Cr), urea, complete blood count $(C B C)$, alanine transaminase $(A L T)$, aspartate transaminase (AST), alkaline phosphatase (ALP), potassium and folate levels were analyzed before, after 5 weeks and after the experiment

Results: Dates vinegar improved the blood concentrations of HbA1c (6.80 \pm 2.34 to $6.17 \pm 2.14$ (\%)), FBS (171.43 \pm 36.74 to $147.56 \pm 38.86 \mathrm{mg} / \mathrm{dL}, p=0.001)$, TC (218.10 \pm 16.9 to $191.14 \pm 14.23 \mathrm{mg} / \mathrm{dL}$, $p<0.001), A L T$ (24.94 \pm 5.03 to $21.88 \pm 5.08 \mathrm{IU} / L, p=0.002)$ and $A L P(264.32 \pm 45.26$ to $257.30 \pm 44.21$ $\mathrm{IU} / \mathrm{L})$ and folate (34.6 \pm 6.6 to $41.7 \pm 6.5 \mathrm{nmol} / \mathrm{L}, p<0.001)$.

Conclusion: Dates vinegar significantly improved the total cholesterol. The other blood biochemical and hematological factors were also improved however; the improvements were not significant.
\end{abstract}

Keywords: Dates vinegar, diabetes, glycated hemoglobin, hyperlipidemia

\begin{abstract}
This is an Open Access article that uses a funding model which does not charge readers or their institutions for access and distributed under the terms of the Creative Commons Attribution License (http://creativecommons.org/licenses/by/4.0) and the Budapest Open Access Initiative (http://www.budapestopenaccessinitiative.org/read), which permit unrestricted use, distribution, and reproduction in any medium, provided the original work is properly credited.

Tropical Journal of Pharmaceutical Research is indexed by Science Citation Index (SciSearch), Scopus, International Pharmaceutical Abstract, Chemical Abstracts, Embase, Index Copernicus, EBSCO, African Index Medicus, JournalSeek, Journal Citation Reports/Science Edition, Directory of Open Access Journals (DOAJ), African Journal Online, Bioline International, Open-J-Gate and Pharmacy Abstracts
\end{abstract}

\section{INTRODUCTION}

Diabetes mellitus (DM) is a universally emergent health complication and is one of the five main causes of mortality and morbidity in many parts of the world [1]. Globally, more than 171 million people were affected by diabetes in 2000 and this figure is projected to reach 366 million by 2030 [2]. Impaired Insulin resistance, insulin secretion and hepatic glucose over-creation are 
possibly related causes of diabetes [3]. Insulin resistance do not merely play a critical role in diabetic problems, but also in dyslipidemia, atherosclerosis and hypertension [4]. Generally, diabetes mellitus is involved in various organ impairment linked disorders including cataract, atherosclerosis, retinopathy, nephropathy and wound restorative impedance [5]. Because of cost of therapy with the various hypoglycemic reagents and insulin commonly used to treat the disease and the unwanted side effects associated with these medications, dietary control is recommended among one of various approaches and techniques required to prevent the diseases.

Results of different studies revealed that some plants and their byproducts are useful in treating chronic diseases such as diabetes, cardiovascular disease and cancer [6]. Acetic acid is considered as main component of vinegar and it is responsible for its biting odor, tart flavor and pungent smell $[7,8]$. Acetate thiokinase, changes the acetic acid to acetyl-coA and is consequently assimilated as citrate. In vitro, the activity of phosphofructokinase is inhibited by citrate but in vivo, citrate increase the repletion of glycogen after meticulous exercise [9]. In 1988, it was revealed for first time that vinegar can be used to cure diabetes. Different animal trials showed that consumption of different doses of acetic acid or vinegar produced fluctuating effects from reduction of hypertension and improvement of glycogen repletion to activation of calcium captivation $[10,11]$. One study showed that white vinegar decreased both insulin levels and postprandial blood glucose [12]. Similarly, another study revealed that intake of apple cider vinegar enhances postprandial insulin sensitivity [13]. Dates vinegar has a variety of flavones and other polyphenols. Dates contain high quantity of anthocyanins, carotenoids and polyphenolic compounds [14]. Extracts of the fruit are used in the prevention of hyperglycemia, hypercholesterolemia, hypertension, and lipoproteins oxidation by reducing the oxidative stress and inflammation on vascular system. The health potentials of dates fruits have been credited to their bioactive compounds, trace elements, vitamin $\mathrm{C}$, tannins, lignins, flavones and other polyphenols especially phenolic acids [1517]. However, no study has explored the beneficial outcomes of dates vinegar consumption against type 2 diabetes.

In this study, the objective was to evaluate the effects of dates vinegar on blood biochemical and hematological parameters in type 2 diabetic subjects.

\section{EXPERIMENTAL}

\section{Experimental samples}

Red date vinegar was provided by Liyang Bask Vinegar Co., Ltd (Hebei, P.R. China). It contained $38.9 \%$ of total polysaccharides. Spectrophotometric technique used for standardization indicated that it contained heavy metal (< $6 \mathrm{ppm})$, arsenic (<1 ppm), cadmium (0.1 $\mathrm{ppm})$ and lead $(<2.5 \mathrm{ppm})$.

\section{Subjects and study design}

Following approval by the Institutional Review Board of Minhag University Lahore (Pakistan), 60type 2 diabetic patients (fasting blood sugar (FBS)>126 mg/dL, 1 antidiabetic drug/daily), aged 30-60 years, 29 females and 26 males weighing between 55 to $75 \mathrm{~kg}$, who visited an AlRashid Clinic Lahore in Pakistan and volunteered to participate in this study were recruited. Those with hepatic, renal, asthmatic or cardiovascular diseases, vinegar intolerance or consumed alcohol were excluded. The patients were randomized into a group (30 patients) that consumed $20 \mathrm{ml}$ of dates vinegar with their normal diet preferably in the morning (or before bedtime) or the placebo group (30 patients) that received $20 \mathrm{ml}$ of honey diluted with water using a randomization scheme with blocks of 5 patients for 10 weeks. Throughout the trial, the participants consumed foods with similar energy levels, carbohydrates, cholesterol, proteins and fats from the beginning to the end.

\section{Collection of blood and biochemical analyses}

The type 2 diabetic patients were exposed to thorough history and clinical evaluation after written informed consent. Blood samples $(5 \mathrm{ml}$ each) were collected at baseline, 5 weeks and at 10 weeks, centrifuged at a speed of $3500 \mathrm{rpm}$ for 15 minutes after allowing to clot and the serum obtained was analyzed for both the biochemical and hematological parameters. Biochemistry automated analyzer (Metro lab 2300 PLUS, Vital Scientific B. V., Netherlands), was used to analyze FBS, triglyceride (TG), high density lipoprotein(HDL), urea and creatinine concentrations according to the manufacturer's instruction while LDL concentration was analyzed using Fried Ewald formula [18]:

$$
\text { LDL-C }=\mathrm{TC}-\mathrm{HDL}-\mathrm{C}-\mathrm{TG} / 5 \quad \ldots \ldots . . \text { Eq. } 1
$$

Hematological automated analyzer (Sysmex XS 1000 ${ }^{\mathrm{TM}}$, Roche, Basel, Switzerland)) was used 
to measure white blood cell (WBC), red blood cell (RBC), mean cell volume (MCV), mean cell hemoglobin $(\mathrm{MCH})$, mean corpuscular hemoglobin concentration (MCHC), hematocrit (HCT) and platelets (PLT)[20,21]. HPLC, composed of Rheodyne injector model 7161 (Rheodyne Co., CA, USA), degasser DG- 440 (Phenomenex Co., Torrance, CA, USA), photodiode-array detector Jasco MD-915 (Tokyo, Japan) and an Agilent UV- detector model 1100, was used to analyze folate and $\mathrm{HbA1c}$ concentrations $[19,20]$.

\section{Statistical analysis}

The Statistical Package for the Social Sciences (SPSS) version 21.0.0 (SPSS Inc., IBM) was used to analyze the data using one-way analysis of variance (ANOVA). Variations in experimental and placebo groups were determined by the paired t-test. All statistical outcomes with a $p$ value of less than 0.05 were deliberated as statistically significant. The values were expressed as mean \pm SD.

\section{RESULTS}

\section{Subject characteristics and diet monitoring}

Of the 60 patients recruited for this study, five participants ( 2 in the date vinegar group and 3 in the placebo group) were excluded from the data analyzed because of protocol variance $(n=3)$ or consent withdrawal $(n=2)$ Figure 1. No serious side effects were observed during the trial. The baseline data for the patients are provided Table 1.

\section{Effect of dates vinegar consumption on various blood biochemical factors}

As from the (Table 3), it can be seen that fasting blood sugar (FBS), cholesterol and LDL showed statistically significant reduction $(P<0.05)$ in dates vinegar group while folate and HDL significantly increased. , however no significant difference was observed in placebo group. $\mathrm{MCH}$ and MCV values decreased, while PLT $(P<0.005)$ enhanced in date vinegar group. Slight variations were also observed in RBC, WBC, hemoglobin, MCHC and HCT (Table 4).

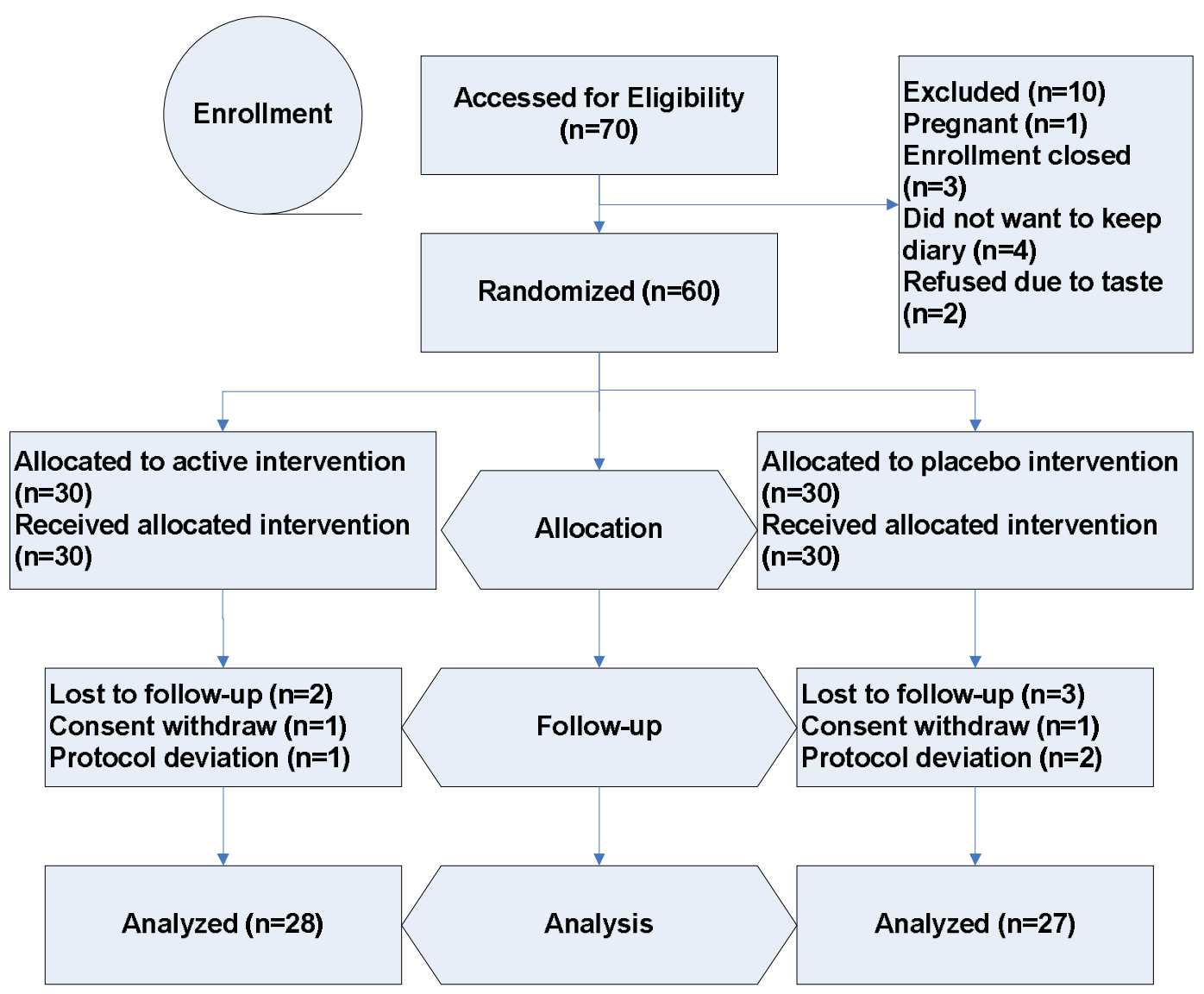

Figure 1: Flow diagram 
Table 1: Baseline characteristics of subjects who completed the intervention trial

\begin{tabular}{|c|c|c|c|}
\hline Variable & $\begin{array}{l}\text { Vinegar group } \\
(n=28) \\
\text { Mean } \pm \text { SD }\end{array}$ & $\begin{array}{l}\text { Placebo group } \\
(\mathrm{n}=27) \\
\text { Mean } \pm \text { SD }\end{array}$ & P-value \\
\hline Age & $46.4 \pm 1.5$ & $48.6 \pm 1.6$ & 0.221 \\
\hline Female/ male (n) & $15 / 13$ & $14 / 13$ & 0.278 \\
\hline BMI (Kg/m2) & $24.2 \pm 0.4$ & $23.3 \pm 0.5$ & 0.389 \\
\hline Systolic BP (mmHg) & $122.1 \pm 1.5$ & $121.0 \pm 1.9$ & 0.463 \\
\hline Diastolic BP (mmHg) & $75.3 \pm 1.4$ & $76.5 \pm 1.6$ & 0.343 \\
\hline Cholesterol (mg/dL) & $218.30 \pm 3.5$ & $216.28 \pm 3.5$ & 0.234 \\
\hline Triglycerides(mg/dL) & $198.25 \pm 4.4$ & $190.20 .1 \pm 4.2$ & 0.014 \\
\hline HDL -C (mg/dL) & $43.4 \pm 4.0$ & $42.5 \pm 5.0$ & 0.178 \\
\hline LDL -C (mg/dL) & $122.4 \pm 4.9$ & $118.4 \pm 4.8$ & 0.032 \\
\hline Urea (mg/dL) & $35.50 \pm 5.6$ & $33.48 \pm 4.5$ & 0.012 \\
\hline Creatinine(mg/dL) & $0.848 \pm 0.4$ & $0.845 \pm 0.7$ & 0.171 \\
\hline Potassium(mEq/L) & $2.98 \pm 0.3$ & $2.91 \pm 0.7$ & 0.247 \\
\hline
\end{tabular}

Values are expressed as mean \pm SD. No significant difference as compared with the initial $(p<0.05)$. BMI; body mass index; BP, blood pressure; HDL-C high-density lipoprotein cholesterol; LDL-C low-density lipoprotein cholesterol VLDL-C very low-density lipoprotein cholesterol.

Table 2: Mean daily intake of energy and selected nutrients at baseline and after intervention

\begin{tabular}{|c|c|c|c|c|c|c|}
\hline \multirow[t]{2}{*}{ Nutrients } & \multicolumn{2}{|c|}{ Vinegar group } & \multicolumn{2}{|c|}{ Placebo group } & \multicolumn{2}{|c|}{ P values } \\
\hline & Before $^{a^{a}}$ & After $^{\text {b }}$ & Before $^{c}$ & After $^{d}$ & $a b$ & cd \\
\hline Energy (kcal) & $1546 \pm 39$ & $1457 \pm 34$ & $1458 \pm 56$ & $1502 \pm 29$ & 0.131 & 0.129 \\
\hline Carbohydrate (g) & $254.9 \pm 6.6$ & $251.5 \pm 5.7$ & $246.7 \pm 6.5$ & $249.5 \pm 5.4$ & 0.451 & 0.376 \\
\hline Protein (g) & $62.2 \pm 4.3$ & $60.6 \pm 4.2$ & $58.7 \pm 2.4$ & $56.1 \pm 2.3$ & 0.261 & 0.158 \\
\hline Total fat (g) & $39.7 \pm 4.6$ & $37.2 \pm 4.7$ & $36.8 \pm 2.6$ & $36.5 \pm 2.7$ & 0.104 & 0.202 \\
\hline Dietary fibers (g) & $17.6 \pm 1.3$ & $23.8 \pm 1.5^{*}$ & $17.5 \pm 1.4$ & $19.9 \pm 1.6$ & 0.001 & 0.511 \\
\hline Saturated Fat (g) & $5.8 \pm 0.8$ & $4.4 \pm 0.5$ & $3.9 \pm 0.5$ & $4.1 \pm 0.7$ & 0.418 & 0.247 \\
\hline Monounsaturated Fat (g) & $7.5 \pm 0.8$ & $7.1 \pm 0.4$ & $6.8 \pm 0.5$ & $6.7 \pm 0.7$ & 0.382 & 0.424 \\
\hline Polyunsaturated Fat (g) & $4.2 \pm 0.6$ & $3.7 \pm 0.8$ & $3.5 \pm 0.8$ & $3.9 \pm 0.6$ & 0.534 & 0.304 \\
\hline$\beta$-carotene $(\mathrm{mg})$ & $2.64 \pm 0.8$ & $3.81 \pm 0.7$ & $2.28 \pm 0.6$ & $2.48 \pm 0.4$ & 0.142 & 0.328 \\
\hline Cholesterol (mg) & $218.2 \pm 6.7$ & $190.4 \pm 6.5^{*}$ & $215.8 \pm 5.7$ & $219.3 \pm 5.9$ & 0.001 & 0.217 \\
\hline
\end{tabular}

Intakes of test products were included in the analysis and estimated from three-day food records. Values are expressed as mean $\pm S D$. *Significant difference as compared with placebo group. ${ }^{a b} \mathrm{P}>0.05 ;{ }^{c d} \mathrm{P}>0.05$.

\section{DISCUSSION}

This is first evidence based study that showed that continuous intake of dates vinegar reduces the HbA1c and FBS levels in patients of type 2 diabetes but increased folate levels in blood. In agreement with our findings, Mahmoodi et al. (2011) revealed that white vinegar consumption by type 2 diabetic patients reduced $\mathrm{HbA} 1 \mathrm{c}$ and improves various biochemical factors like total cholesterol and LDL as compared to placebo.
Similarly, one researcher showed that regular consumption of vinegar reduced the $\mathrm{HbA1c}$ $(0.16 \%)$ concentration in experimental subjects as compared to control [21]. In the small intestine, acetic acid, major component of dates vinegar, stalled the starch molecules digestion completely via blocking the activity of disaccharides and also reduces uptake of glucose through muscle performance. Intake of 0.2 per 100 grams of acetic acid decreased accretion of xylulose-5- phosphate (Xu-5-P) in 
Table 3: Effects of dates vinegar consumption on various biochemical factors

\section{Dates vinegar group}

\section{Placebo group}

\begin{tabular}{|c|c|c|c|c|c|c|}
\hline$\overline{\text { Variable }}$ & Baseline & After 5 weeks & After 10 weeks & Baseline & After 5 weeks & After 10 weeks \\
\hline HbA1c (\%) & $6.80 \pm 2.34$ & $6.54 \pm 2.31$ & $6.17 \pm 2.14$ & $6.55 \pm 3.25$ & $6.67 \pm 3.65$ & $6.72 \pm 4.16$ \\
\hline FBS (mg/dL) & $171.43 \pm 3.74$ & $159.36 \pm 4.61$ * & * $147.56 \pm 3.86$ * & $164.43 \pm 2.64$ & $169.51 \pm 2.73$ & $168.65 \pm 2.23$ \\
\hline Cholesterol(mg/dL) & $218.10 \pm 3.9$ & $198.12 \pm 3.11$ * & ${ }^{*} 191.14 \pm 3.7^{*}$ & $213.1 \pm 4.6$ & $209.3 \pm 4.8$ & $211.4 \pm 4.9$ \\
\hline HDL (mg/dL) & $41.7 \pm 4.7$ & $44.1 \pm 4.6^{*}$ & $47.5 \pm 4.5^{*}$ & $40.5 \pm 3.5$ & $42.3 \pm 3.3$ & $41.4 \pm 3.9$ \\
\hline LDL(mg/dL) & $117.14 \pm 3.6$ & $102.16 \pm 3.5^{\star}$ & $97.15 \pm 3.7^{*}$ & $114.16 \pm 2.6$ & $118.20 \pm 2.2$ & $121.12 \pm 2.4$ \\
\hline Triglyceride(mg/dL & $213.30 \pm 4.4$ & $211.21 \pm 4.5$ & $207.17 \pm 4.7$ & $209.4 \pm 5.7$ & $213.1 \pm 5.6$ & $214.5 \pm 5.8$ \\
\hline VLDL(mg/dL) & $46.90 \pm 3.7$ & $46.10 \pm 3.6$ & $45.44 \pm 3.5$ & $43.64 \pm 3.8$ & $43.62 \pm 3.7$ & $44.48 \pm 3.9$ \\
\hline Urea $(\mathrm{mg} / \mathrm{dL})$ & $37.11 \pm 4.4$ & $35.13 \pm 4.4$ & $34.16 \pm 4.2$ & $39.86 \pm 3.4$ & $38.5 \pm 3.3$ & $39.3 \pm 3.4$ \\
\hline Creatinine (mg/dL) & $0.843 \pm 3.6$ & $0.841 \pm 3.7$ & $0.839 \pm 3.4$ & $0.848 \pm 5.6$ & $0.850 \pm 5.9$ & $0.849 \pm 6.1$ \\
\hline Folate (nmol/ L) & $34.6 \pm 6.6$ & $37.6 \pm 6.7^{*}$ & $41.7 \pm 6.5^{\star}$ & $35.4 \pm 5.8$ & $37.6 \pm 5.6$ & $38.1 \pm 5.7$ \\
\hline ALT (IU/L) & $24.94 \pm 5.03$ & $22.93 \pm 4.08$ & $21.88 \pm 5.08$ & $25.76 \pm 6.06$ & $24.85 \pm 6.04$ & $25.78 \pm 6.09$ \\
\hline AST (IU/L) & $30.86 \pm 4.09$ & $29.44 \pm 3.06$ & $28.53 \pm 3.08$ & $31.98 \pm 4.07$ & $31.99 \pm 4.01$ & $31.77 \pm 4.00$ \\
\hline ALP (IU/L) & $264.32 \pm 5.26$ & $260.36 \pm 4.28$ & $259.30 \pm 4.21$ & $267.54 \pm 3.32$ & $268.48 \pm 3.41$ & $271.24 \pm 3.26$ \\
\hline Potassium (mEq/ L) & $2.98 \pm 1.7$ & $2.73 \pm 1.8$ & $2.85 \pm 1.6$ & $2.91 \pm 1.5$ & $2.86 \pm 1.3$ & $2.81 \pm 1.4$ \\
\hline
\end{tabular}

Table 4: Effects of dates vinegar on various hematological factors

\begin{tabular}{|c|c|c|c|c|c|c|}
\hline \multirow[b]{2}{*}{ Variable } & \multirow[b]{2}{*}{ Baseline } & \multicolumn{2}{|c|}{ Dates Vinegar group } & \multirow[b]{2}{*}{ Baseline } & \multicolumn{2}{|l|}{ Placebo group } \\
\hline & & After 5 weeks & After 10 weeks & & After 5 weeks & After 10 weeks \\
\hline WBC(n/ml) & $8923.04 \pm 1217$ & $9245.25 \pm 1085$ & $9515.38 \pm 1510$ & $9014.05 \pm 1432$ & $9145.1674 \pm 1241$ & $9168.1811 \pm 1123$ \\
\hline $\begin{array}{l}\text { RBC (mil/ml) } \\
\text { Hemoglobin } \\
\text { (g/dl) }\end{array}$ & $\begin{array}{l}5.512 \pm 0.458 \\
14.11 \pm 3.26\end{array}$ & $\begin{array}{l}5.524 \pm 0.44 \\
14.45 \pm 3.31\end{array}$ & $\begin{array}{l}5.608 \pm 0.42 \\
14.88 \pm 3.46\end{array}$ & $\begin{array}{l}5.413 \pm 0.31 \\
14.25 \pm 2.43\end{array}$ & $\begin{array}{l}5.418 \pm 0.331 \\
14.30 \pm 2.52\end{array}$ & $\begin{array}{l}5.425 \pm 0.32 \\
14.33 \pm 2.21\end{array}$ \\
\hline HCT (\%) & $44.34 \pm 4.542$ & $44.84 \pm 4.49$ & $45.25 \pm 4.51$ & $45.12 \pm 4.33$ & $45.74 \pm 4.341$ & $45.98 \pm 4.36$ \\
\hline $\operatorname{MCV}(f \mathrm{l})$ & $87.11 \pm 5.304$ & $84.65 \pm 5.43$ & $80.44 \pm 5.53$ & $88.04 \pm 4.32$ & $88.24 \pm 4.344$ & $88.79 \pm 4.35$ \\
\hline MCH (pg) & $26.55 \pm 3.5$ & $24.86 \pm 3.1$ & $21.45 \pm 3.3$ & $25.31 \pm 3.8$ & $25.44 \pm 3.4$ & $25.02 \pm 3.2$ \\
\hline MCHC (\%) & $28.67 \pm 1.44$ & $28.88 \pm 1.32$ & $29.35 \pm 1.43$ & $29.25 \pm 1.34$ & $29.41 \pm 1.25$ & $29.36 \pm 1.47$ \\
\hline PLT (n/ml) & $256076 \pm 31584$ & $284742 \pm 45264$ & $318307 \pm 62581$ & $258934 \pm 34273$ & $256831 \pm 41156$ & $254365 \pm 49187$ \\
\hline
\end{tabular}

Variables are expressed as mean \pm SD. No significant difference as compared with the baseline $(p<0.05)$

RBC; Red blood cells, WBC; White blood cells, HCT; Hematocrit, MCHC; Mean cell hemoglobin concentration, $\mathrm{MCV}$; Mean cell volume, MCH; Mean cell hemoglobinPLT; Platelets

liver and activity of phosphofructokinase-1 (PFK1 ) in skeletal muscles [9]. Acetic acid also slows down the rate of gastric emptying [22]. Enhancement of free fatty acids in subjects having impaired glucose tolerance, presupposes that insulin resistance connected with enhanced free fatty acids befalls earlier the inception of hyperglycemia [23]. Results of one trial conducted in non-diabetic subjects reveled that reduced glucose exploitation in muscles was connected with severe raise of free fatty acids [24]. Some experiments also showed association among insulin resistance and levels of free fatty acids $[25,26]$. During the manifestation of insulin resistance, free fatty acids in the form of triglycerides are stored in heart, liver, pancreas 
and muscle. Particularly, agents that reduced enhanced levels of free fatty acids, like thiazolidinedione's, have been exposed to upregulate insulin sensitivity in liver, adipose tissue and muscle. As, outcomes of our study showed that regular consumption of dates vinegar reduces lipid profile parameters hence, we can say that dates vinegar might be involved in improving insulin sensivity by reducing the free fatty acids levels.

High level of fructose and fiber content in dates might be interconnected with reduced level of glycemic index (Gl). The results of one study revealed that regular consumption of rutab dates enhanced the $\mathrm{Gl}$ and suggested that high content of rutab dates hydration affects gastric emptying, insulin secretion or intestinal absorption [27]. Similarly, results of various studies showed that ingestion of dietary fibers applies physiological effects on the small intestine and stomach that curb postprandial glycemic responses, comprising adjourning small bowel passage and modifying myoelectric commotion of gastrointestinal way, suspending gastric emptying lessening the availability of $\alpha$ amylase to its substrate because of enhanced gut contents viscosity, and decreasing diffusion of glucose via callous water layer gel forming and enhanced viscosity chattels of fibers are primarily accountable for its glycemic effect. Because of reformed levels of incretin, soluble fibers prolonged the carbohydrates absorption in intestine along with enhanced levels of glucagonlike peptide 1 (GLP1) [28-30]. Soluble dietary fibers also affect the uptake mechanisms of glucose via periphery, comprising enhanced skeletal muscle manifestation of the insulinresponsive glucose transporter type 4 (GLUT-4), which increases skeletal muscle upregulation, enhances sensitivity of insulin and regularizes blood glucose [31]. Several fatty acids in humans stimulate the manifestation of peroxisome proliferator-activated receptor-y (PPARG), which enhances levels of adipocyte GLUT-4 [32]. During metabolic disorders the levels of hepatic enzymes like ALT and AST increased and thus resulted in atherosclerosis [33]. In our study the levels of ALT, AST and ALP reduced at the end of trial in the experimental group. While, the results of one study showed that the activity of liver AST and ALP in the animals treated with the vinegar decreased and elevated, respectively [34].

Current results showed slight variations in the number of RBC, WBC count and its allied indices like $\mathrm{Hb}, \mathrm{MCHC}, \mathrm{MCV}, \mathrm{MCH}$ and platelets. Total $\mathrm{RBC}$ and $\mathrm{Hb}$ along with platelets increased at the end of study. While, results of one study showed that no substantial variations were observed in these variables after ingestion of vinegar [35]. The increase in total RBC and $\mathrm{Hb}$ might be due to high content of folate in dates.

In summary, this study indicates that date vinegar improves the concentration of $\mathrm{HbA} 1 \mathrm{c}$, FBS and lipid profile, and/or potentiates the capacity of anti-glycemic remedies in type 2 diabetic subjects. The limitations of this study include several proposed molecular mechanisms through which date vinegar may exert its effect were not measured directly. More studies are required to substantiate these preliminary outcomes. During the trial, neither deformity of kidney or liver functions nor adversative outcomes were noted. As regular consumption of dates vinegar $(20 \mathrm{~mL})$ for 10 weeks in diabetic patients was testified not to cause side effects, dates vinegar consumption is supposed to be safe in type 2 diabetic patients.

\section{CONCLUSION}

Conclusively based on the present outcomes, it can be decided that dates vinegar is beneficial as a therapeutic agent in diabetes, without producing side effects. Intake of $20 \mathrm{~mL}$ of dates vinegar on daily basis was adequate to attain these effects. Dates vinegar might be considered helpful for precluding metabolic syndrome by reducing diabetes and its related complications. However, more experiments are required to discover both its disadvantages and advantages as a tool for directing type 2 diabetes.

\section{DECLARATIONS}

\section{Acknowledgements}

This work was supported by funds from the National R \& D Program (Grant Number: 2016YFD0400303-1), National High Technology Research and Development Program 863 (Grant Number: 2013AA102203) and Jiangsu provincial major project on natural science for universities (Grant Number: 12KJA55000)

\section{Conflict of interest}

The authors declare that they have no conflict of interest with regard to this work.

\section{Authors' contribution}

We declare that this work was performed by the authors named in this article, and all liabilities pertaining to claims relating to the content of this article will be borne by the authors. All the 
authors listed in this manuscript contributed equally, and read and approved the manuscript.

\section{REFERENCES}

1. Gispen WH, Biessels GJ. Cognition and synaptic plasticity in diabetes mellitus. Trends Neurosci 2000; 23: $542-549$

2. Wild S, Roglic G, Green A, Sicree R, King H. Global prevalence of diabetes: estimates for the year 2000 and projections for 2030. Diabetes care 2004; 27 : 1047-1053.

3. Cerf ME. Beta cell dysfunction and insulin resistance. Frontiers in endocrinology 2013;4: 37.

4. Bornfeldt KE, Tabas I. Insulin resistance, hyperglycemia, and atherosclerosis. Cell Metab 2011;14: 575-585

5. Peters A, Laffel L, Group ADATW. Diabetes care for emerging adults: recommendations for transition from pediatric to adult diabetes care systems: a position statement of the American Diabetes Association, with representation by the American College of Osteopathic Family Physicians. Diabetes care 2011;34: 2477-2485.

6. Sofowora A, Ogunbodede E, Onayade $A$. The role and place of medicinal plants in the strategies for disease prevention. Afr $J$ Tradit Complement AlternMed 2013;10: 210-229.

7. Cortesia $C$, Vilcheze $C$, Bernut A. Acetic acid, the active component of vinegar, is an effective tuberculocidal disinfectant. M Bio 2014;5: 13-14.

8. Ali Z, Wang Z, Amir RM, Younas S, Wali A, Adowa N. Potential Uses of Vinegar as a Medicine and Related in vivo Mechanisms. IJVNR 2017; 1:1-12.

9. Fushimi $T$, Tayama K, Fukaya M. The efficacy of acetic acid for glycogen repletion in rat skeletal muscle after exercise. Int J Sports Med 2002;23: 218-222.

10. Fushimi T, Tayama K, Fukaya M. Acetic acid feeding enhances glycogen repletion in liver and skeletal muscle of rats. J Nutr 2001:131: 1973-1977.

11. Davalos A, Bartolome B, Gomez-Cordoves C. Antioxidant properties of commercial grape juices and vinegars. Food Chem 2005;93: 325-330.

12. Ostman E, Granfeldt Y, Persson L, Bjorck I. Vinegar supplementation lowers glucose and insulin responses and increases satiety after a bread meal in healthy subjects. Eur J Clin Nutr 2005;59: 983.

13. White $A M$, Johnston CS. Vinegar ingestion at bedtime moderates waking glucose concentrations in adults with well-controlled type 2 diabetes. Diabetes Care 2007;30: 2814-2815.

14. Amira EA, Behija SE, Beligh $M$. Effects of the ripening stage on phenolic profile, phytochemical composition and antioxidant activity of date palm fruit. J Agric Food Chem 2012:60: 10896-10902.

15. Al-Shoaibi Z, Al-Mamary MA, Al-Habori MA, Al-Zubairi AS, Abdelwahab SI. In vivo antioxidative and hepatoprotective effects of palm date fruits (Phoenix dactylifera). Int J Pharmacol 2012;8: 185-191.

16. Vayalil PK. Date fruits (Phoenix dactylifera Linn): an emerging medicinal food. Crit Rev Food Sci Nutr 2012;52: 249-271.

17. Ali Z, Ma H, Ayim I, Wali A. Efficacy of new beverage made of dates vinegar and garlic juice in improving serum lipid profile parameters and inflammatory biomarkers of mildly hyperlipidemic adults: $A$ double-blinded, randomized, placebo-controlled study. J Food Biochem 2018; e12545.

18. Friedewald WT, Levy RI, Fredrickson DS. Estimation of the concentration of low-density lipoprotein cholesterol in plasma, without use of the preparative ultracentrifuge. Clin Chem 1972;18: 499-502.

19. Davis, J E, Mcdonald J M, Jarett L. A high-performance liquid chromatography method for hemoglobin A1c. Diabetes 1978; 27: 102-107.

20. Kalmbach $R$, Paul L, Selhub J. Determination of unmetabolized folic acid in human plasma using affinity HPLC. Am J Clin Nutr 2011: 94: 343-347.

21. Johnston $S$, White $A$, Kent M. Preliminary evidence that regular vinegar ingestion favorably influences hemoglobin $A 1 C$ values in individuals with type 2 diabetes mellitus. Diabetes Res Clin Pact 2009; 84:1517.

22. Hlebowicz J, Darwiche G, Bjorgell O, Almer LO. Effect of apple cider vinegar on delayed gastric emptying in patients with type 1 diabetes mellitus: a pilot study. BMC Gastroenterol 2007;7: 46.

23. Delarue J, Magnan C. Free fatty acids and insulin resistance. Curr Opin Clin Nutr Metab Care 2007;10: 142-148.

24. Boden G. 450besity, insulin resistance and free fatty acids. Curr Opin Endocrinol Diabetes Obes 2011;18: 139.

25. Shah $P$, Vella A, Basu A. Effects of free fatty acids and glycerol on splanchnic glucose metabolism and insulin extraction in nondiabetic humans. Diabetes 2002;51: 301-310.

26. Hodson L, Bickerton AS, McQuaid SE. The contribution of splanchnic fat to VLDL triglyceride is greater in insulin-resistant than insulin-sensitive men and women: studies in the postprandial state. Diabetes 2007;56: 2433-2441.

27. Miller C, Dunn E, Hashim I. The glycaemic index of dates and date/yoghurt mixed meals. Are dates the candy that grows on trees'? Eur J Clin Nutr 2003;57: 427.

28. Schulze MB, Liu S, Rimm EB, Manson JE, Willett WC, Hu FB. Glycemic index, glycemic load, and dietary fiber intake and incidence of type 2 diabetes in younger and middle-aged women. Am J Clin Nutr 2004;80: 348356.

29. Brownlee IA. The physiological roles of dietary fibre. Food Hydrocoll 2011;25: 238-250.

30. Leclere CJ, Champ M, Boillot J, et al. Role of viscous guar gums in lowering the glycemic response after a solid meal. Am J Clin Nutr 1994;59: 914-921.

31. Song YJ, Sawamura M, Ikeda K, Igawa S, Yamori $Y$. Soluble Dietary Fibre Improves Insulin Sensitivity by Increasing Muscle Glut-4 Content in Stroke-Prone Spontaneously Hypertensive Rats. Clin Exp Pharmacol Physiol 2000;27: 41-45.

32. Park KS, Ciaraldi TP, Lindgren K. Troglitazone effects on gene expression in human skeletal muscle of type II diabetes involve up-regulation of peroxisome proliferator-activated receptor-y. J Clin Endocrinol Metabol 1998;83: 2830-2835.

33. Setorki M, Asgary S, Eidi A, Hazaei M. Acute effects of vinegar intake on some biochemical risk factors of atherosclerosis in hypercholesterolemic rabbits. Lipids Health Dis 2010;9: 10.

34. Mohamed M, Mohamed SM, Mohamed KA. The effect of cider vinegar on some nutritional and physiological parameters in mice. J Egypt Public Health Assoc 2001 76:17-36

35. Mahmoodi M, Hosseini-zijoud S-M, Nabati S. The effect of white vinegar on some blood biochemical factors in type 2 diabetic patients. I Diabetes Endocrinol 2013;4: 